\title{
In-line Raman spectroscopy for gate-all-around nanosheet device manufacturing
}

\author{
Daniel Schmidt $\odot,{ }^{\text {a, } *}$ Curtis Durfee, ${ }^{a}$ Juntao Li, ${ }^{\text {a }}$ Nicolas Loubet, ${ }^{a}$ \\ Aron Cepler, ${ }^{b}$ Lior Neeman ${ }^{c}{ }^{c}$ Noga Meir, ${ }^{c}$ Jacob Ofek, ${ }^{c}$ \\ Yonatan Oren, ${ }^{c}$ and Daniel Fishman ${ }^{c}$ \\ ${ }^{a}$ IBM Research, Albany, New York, United States \\ ${ }^{b}$ Nova Inc., Fremont, California, United States \\ ${ }^{c}$ Nova Ltd., Rehovot, Israel
}

\begin{abstract}
In-line Raman spectroscopy for compositional and strain metrology throughout frontend-of-line (FEOL) manufacturing of next-generation gate-all-around nanosheet field-effect transistors is presented. Thin and alternating layers of fully strained pseudomorphic $\mathrm{Si}_{(1-x)} \mathrm{Ge}_{x}$ and $\mathrm{Si}$ were grown epitaxially on a Si substrate and subsequently patterned. Intentional strain variations were introduced by changing the Ge content $(x=0.25,0.35,0.50)$. Polarization-dependent in-line Raman spectroscopy was employed to characterize and quantify the strain evolution of $\mathrm{Si}$ and $\mathrm{Si}_{(1-x)} \mathrm{Ge}_{x}$ nanosheets throughout FEOL processing by focusing on the analysis of $\mathrm{Si}-\mathrm{Si}$ and Si-Ge optical phonon modes. To evaluate the accuracy of the Raman metrology results, strain reference data were acquired by non-destructive high-resolution x-ray diffraction and from destructive lattice deformation maps using precession electron diffraction. It was found that the germanium-alloy composition as well as $\mathrm{Si}$ and $\mathrm{Si}_{(1-x)} \mathrm{Ge}_{x}$ strain obtained by Raman spectroscopy are in very good agreement with reference metrology and follow trends of previously published simulations. $\odot$ The Authors. Published by SPIE under a Creative Commons Attribution 4.0 International License. Distribution or reproduction of this work in whole or in part requires full attribution of the original publication, including its DOI. [DOI: 10.1117/1.JMM.21.2.021203]
\end{abstract}

Keywords: Raman spectroscopy; strain; stress; gate-all-around; nanosheet field-effect transistor.

Paper 21077SS received Sep. 17, 2021; accepted for publication Jan. 11, 2022; published online Jan. 27, 2022.

\section{Introduction}

Nanosheet gate-all-around (GAA) field effect-transistors (FETs) will be the next-generation device architecture replacing finFET technology either next year or in $2023 .{ }^{1}$ The new transistor type is an evolutionary step from finFETs with better performance due to superior electrostatics and short channel control, for example. ${ }^{2}$ A nanosheet GAAFET may be viewed, in a simplistic manner, as a finFET rotated on its side, where the fin is now a horizontal nanosheet with a gate wrapped around the entire perimeter. Typically, the device comprises three or more vertically stacked individual horizontal nanosheets, which serve as channels. This is accomplished by first growing alternating pseudomorphic $\mathrm{Si}_{(1-x)} \mathrm{Ge}_{x}$ and $\mathrm{Si}$ layers on a Si substrate. Here, a perfect crystal quality is critical to achieve defect free, biaxially strained $\mathrm{Si}_{(1-x)} \mathrm{Ge}_{x}$, and strain-free $\mathrm{Si}$ single crystalline layers. This multilayer stack is then patterned in both in-plane orientations to define channel length and width. Usually, the $\mathrm{Si}_{(1-x)} \mathrm{Ge}_{x}$ sheets are sacrificial and removed soon after source/drain epitaxy leaving the Si sheets as transport channels. ${ }^{2}$ To pursue optimum transistor performance, it is desirable to increase charge carrier mobility through tensile and compressive strain for nFET and pFET, respectively. The electron mobility in GAA nFET Si channels is intrinsically higher than that of finFETs due to the sheet orientation and some residual tensile strain after patterning. However, the advantages for nFET negatively impact the pFET hole mobility. Additionally, as the transistor volume shrinks due to aggressive pitch scaling and three-dimensional stacking, traditional external channels stressors are often not very effective. In addition, integration schemes of horizontal nanosheets wrapped with high-k/metal gate

*Address all correspondence to Daniel Schmidt, schmidt@ibm.com 
materials create many unconstrained free surfaces throughout the patterning process, leading to natural relaxation. Overcoming these challenges requires either complex and costly manufacturing schemes or innovative strain engineering approaches. ${ }^{3,4}$

No matter which integration scheme is pursued, the complex manufacturing process, particularly related to the channels and their immediate environment, requires tight specification limits and hence additional, more advanced, and novel in-line metrology capabilities compared to previous transistor architectures. Specifically, monitoring and controlling strain at such small scales and within intricate nanoscale device structures is a new challenge and a suitable nondestructive and fast in-line technique is critical for successful research and development and manufacturing. Relying on destructive imaging and in-line electrical test results is not a viable option for competitive development or efficient and economical high-volume manufacturing.

So far, quantitative strain measurements for GAAFETs are relying on scanning transmission electron microscope (STEM)-based techniques. The strain is measured by either nanobeam diffraction, geometrical phase analysis, or most precisely by precession electron diffraction (PED). Beam diameters of $\sim 5 \mathrm{~nm}$ allow mapping of channel strain even in aggressively scaled FETs with channel lengths of $<20 \mathrm{~nm} \cdot{ }^{4-7}$ However, TEM techniques are time-consuming, destructive, and require sample preparation, which may influence strain characteristics.

Non-destructive high-resolution $\mathrm{x}$-ray diffraction $(\mathrm{XRD})$ techniques usually require a large spot size and long measurement times due to the limited brightness of available fab tools sources. Furthermore, the signal-to-noise ratio depends on the interaction volume, which makes measurements of patterned samples with thin films and low areal density difficult. With continued scaling and hence smaller pitches, larger reciprocal space maps (RSMs) are required to capture enough information for strain evaluation, which additionally affects the measurement time. Schulze et al. reported RSMs in the vicinity of the asymmetric (113) Bragg reflection parallel and perpendicular to patterned fins. While they concluded that the multilayer fins are fully strained along the fin direction and partially relaxed perpendicular to it, no quantitative results are presented. ${ }^{8}$

In this work, for the first time, in-line Raman spectroscopy is presented for non-destructive strain metrology at seven different process steps throughout the front-end-of-line (FEOL) manufacturing cycle of GAAFETs. The strain evolution is tracked for three experimental scenarios with intentionally different initial strain conditions. Here, the focus is put on nFET device baseline monitoring. The determined strain is compared to reference metrology and discussed with respect to recently published simulation results. ${ }^{5,6}$

\section{Experimental Details}

For this study, nanosheet GAA nFETs were manufactured in a very similar manner to what was published earlier. ${ }^{2}$ The nanosheet stack is composed of alternating $\mathrm{Si}_{(1-x)} \mathrm{Ge}_{x}$ and $\mathrm{Si}$ layers, three each, grown on a Si substrate. The only intentional variation introduced in the manufacturing process was during nanosheet stack epitaxy. The experiment comprises three different split conditions where the sacrificial $\mathrm{Si}_{(1-x)} \mathrm{Ge}_{x}$ nanosheet layers are pseudomorphically grown with varying Ge content (nominally $x=0.25,0.35,0.50$ ). This introduces different initial biaxial strain conditions since the compressive strain in defect-free pseudomorphic $\mathrm{Si}_{(1-x)} \mathrm{Ge}_{x}$ layers increases monotonically with $x$ due to the increasing lattice mismatch with $\mathrm{Si}$.

In-line polarized Raman spectroscopy measurements were carried out on a commercially available $300 \mathrm{~mm}$ fab tool (Nova ELIPSON) after seven different processing steps throughout the FEOL manufacturing cycle: (i) post nanosheet stack epitaxy, (ii) post fin patterning, (iii) post dummy gate patterning, (iv) post fin recess (second fin patterning), (v) post source/drain (S/D) epitaxy, (vi) post dummy gate removal, and (vii) post channel release. Multiple targets with varying fin and gate critical dimensions (CDs) were measured for 21 fields across the wafer to evaluate the dimensional dependence of strain as well as the across wafer characteristics. The experimental settings are such that most if not all $\mathrm{Si}$ and $\mathrm{Si}_{(1-x)} \mathrm{Ge}_{x}$ nanosheets contribute to the Raman scattering response. Hence results must be understood as average characteristics of all probed sheets over an array of multiple fins and gates where present. 
After the nanosheet stack epitaxy in-line high-resolution $\omega-2 \theta$ XRD scans (Bruker JVX7300LSI) around the (004) Bragg reflection were analyzed to determine sheet thickness and Ge content. Data were acquired at five different locations across the wafer. Additionally, asymmetric (113) glancing exit RSMs were acquired to confirm defect-free pseudomorphic growth of the $\mathrm{Si}_{(1-x)} \mathrm{Ge}_{x}$ sheets. Due to the long measurement times, only one RSM was acquired per sample. With the confirmation of defect-free single crystalline films, the in-plane sheet strain can then be calculated based on the Ge content-dependent alloy lattice mismatch. ${ }^{9}$

Furthermore, after fin etch, asymmetric (113) glancing exit RSMs were acquired parallel and perpendicular to the fin grating to evaluate the strain post patterning. Due to the larger spot size of the XRD tool, only one target with a fin CD of $40 \mathrm{~nm}$ could be measured, which is adjacent to the targets measured by Raman spectroscopy. The in-plane strain can be determined based on the position of the intensity envelope maximum in the $Q_{x}$ direction. ${ }^{8,10}$ Additionally, in-plane $\mathrm{Si}_{(1-x)} \mathrm{Ge}_{x}$ sheet strain based on destructive lattice deformation maps was calculated. The maps were determined from nano-beam PED patterns acquired with a convergence semi-angle of $2.5 \mathrm{mrad}$, a beam precession at $200 \mathrm{~Hz}$ at an angle of $0.35 \mathrm{deg}$, and an exposure time of $50 \mathrm{~ms}$. The unstrained Si reference is taken from the fin base away from the nanosheet stacks. ${ }^{7}$

\section{Raman Spectroscopy}

Raman spectroscopy has long been shown to be an excellent non-destructive technique to measure stress in Si-based semiconductor devices. ${ }^{10-12}$ It relies on inelastic scattering of incident photons, which interact with vibrational energy states of the sample system. The scattered light can be either lower (Stokes Raman scattering) or higher (anti-Stokes Raman scattering) in energy and the magnitude of the shift corresponds to the specific vibrational modes. The energy shift depends strongly on the exact geometry and length of atomic bonds and hence the Raman scattering response is a unique signature of composition and structure. Therefore, analyzing the Raman fingerprint of a sample allows for determination of characteristics such as composition, strain, and crystallinity, for example.

From an experimental point of view, the choice of excitation energy along with polarization settings within the incident and scattered beam paths are most critical considerations. Depending on the sample under investigation, the incident wavelength determines penetration depth and Raman scattering efficiency. For example, the penetration depth for an excitation wavelength of $400 \mathrm{~nm}$ in $\mathrm{SiGe}_{25}$ is about $30 \mathrm{~nm}$, while $450 \mathrm{~nm}$ penetrate already around $200 \mathrm{~nm}$ into the film. Hence, shorter wavelengths probe in the vicinity of the surface, and with longer wavelengths, information from deeper sample regions can be accessed. The Raman scattering efficiency and therefore the Raman peak intensity strongly depends on the composition of $\mathrm{Si}_{(1-x)} \mathrm{Ge}_{x}$, for instance. Shorter wavelengths are desired for silicon-rich and longer wavelengths for germanium-rich alloys, respectively. ${ }^{13}$

Equally important is polarization control when measuring single crystalline films or anisotropic samples, for instance. Yoo et al. ${ }^{14}$ have shown the polarization-dependence of Raman signals from bare Si wafers: the intensity as well as the peak position depend on the crystallographic orientation. Particularly important are polarization-dependent Raman measurements when the samples are patterned. Figure 1(a) shows how the Raman scattering intensities as a function of wavenumber depend on the polarization orientation. Here, the sample is a stack of alternating pseudomorphic $\mathrm{SiGe}_{35}$ and Si layers on a Si substrate post patterning [Fig. 1(b)]. A gradual transition of the polarization direction from parallel to perpendicular to the fins reveals successively dramatic changes. The peak intensity around $510 \mathrm{~cm}^{-1}$ steadily decreases while the position remains constant. The peak around $520 \mathrm{~cm}^{-1}$ is slightly increasing in intensity and shifting toward higher wavenumbers.

The observed far-field Raman scattering of such patterned $\mathrm{Si}$ and $\mathrm{Si}_{(1-x)} \mathrm{Ge}_{x}$ nanosheet stacks is a complex response depending on electric field localizations, polarization-dependent selection rules for backscattering from the (001) surface (relaxed due to patterning), and three different optic phonon modes $\left(\mathrm{TO}_{1}, \mathrm{TO}_{2}\right.$, and LO). ${ }^{12,15}$ Rigorous electromagnetic simulations show that when the light polarization is parallel to the fins, the electric field is concentrated within the fin with a dominant LO phonon. In this measurement configuration, the response is dominated by 


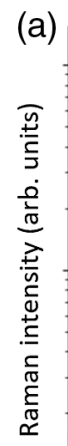

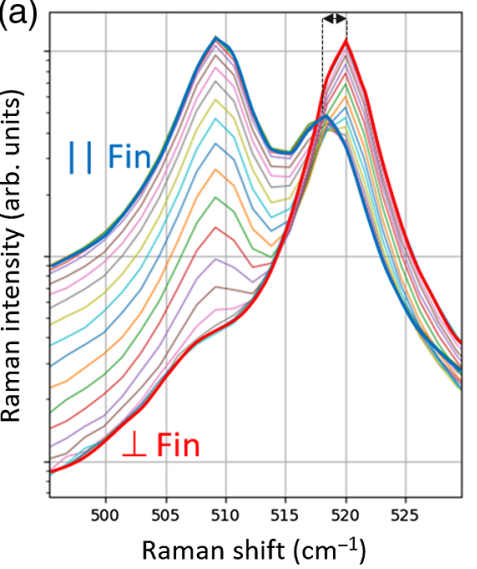

(b)

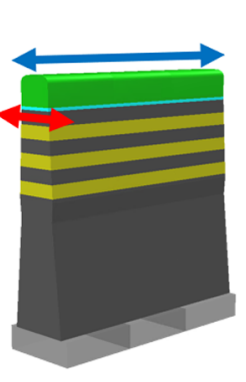

(c)

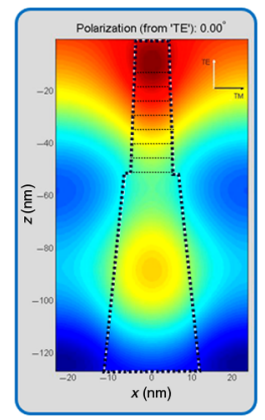

(d)

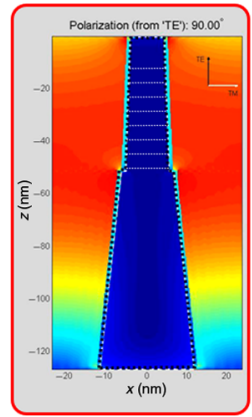

Fig. 1 (a) Raman scattering intensity as a function of wavenumber and polarization orientation for a patterned stack of alternating $\mathrm{SiGe}_{35}$ and Si layers on a Si substrate. The polarization direction is gradually rotated from parallel (blue) to perpendicular (red) to the fins; (b) schematic of the fin with the multilayer nanosheet stack, the arrows indicate parallel (blue) and perpendicular (red) configurations; electromagnetic simulations for (c) parallel and (d) perpendicular polarizations illustrate the electric field distribution.

the properties of the multilayers along the fins [Fig. 1(c)]. With light polarization in the orthogonal direction (perpendicular to the fins), the electric field is mostly excluded from the fin [Fig. 1(d)] and the three optic phonon modes related to the nanosheet stack contribute similarly to Raman scattering from all parts of the sheets. A detailed strain decomposition is possible by analyzing multiple polarization-dependent metrology configurations but beyond the scope of this work. In general, the Raman signal depends quadratically both on the illumination and collection near-field couplings. With the resulting fourth power dependence on the local field distributions, the simulations indicate that polarization-dependent measurements are highly selective.

Figure 2 shows Raman spectra acquired before patterning for the three sample scenarios with varying $\mathrm{Ge}$ content that are considered for this study $\left(\mathrm{SiGe}_{25}, \mathrm{SiGe}_{35}\right.$, and $\left.\mathrm{SiGe}_{50}\right)$. Additionally, a representative high-angle annular dark-field (HAADF) STEM cross-section image and qualitative elemental maps of $\mathrm{Si}$ and $\mathrm{Ge}$ for the $\mathrm{SiGe}_{35}$ sample post fin patterning are presented. The Raman spectra are dominated by optic phonons involving Ge-Ge, Si-Ge, and Si-Si stretching motions at around 300,400 , and $500 \mathrm{~cm}^{-1}$, respectively. In addition to these peaks, each spectrum contains some more weak features between 425 and $475 \mathrm{~cm}^{-1}$. Careful examination of the

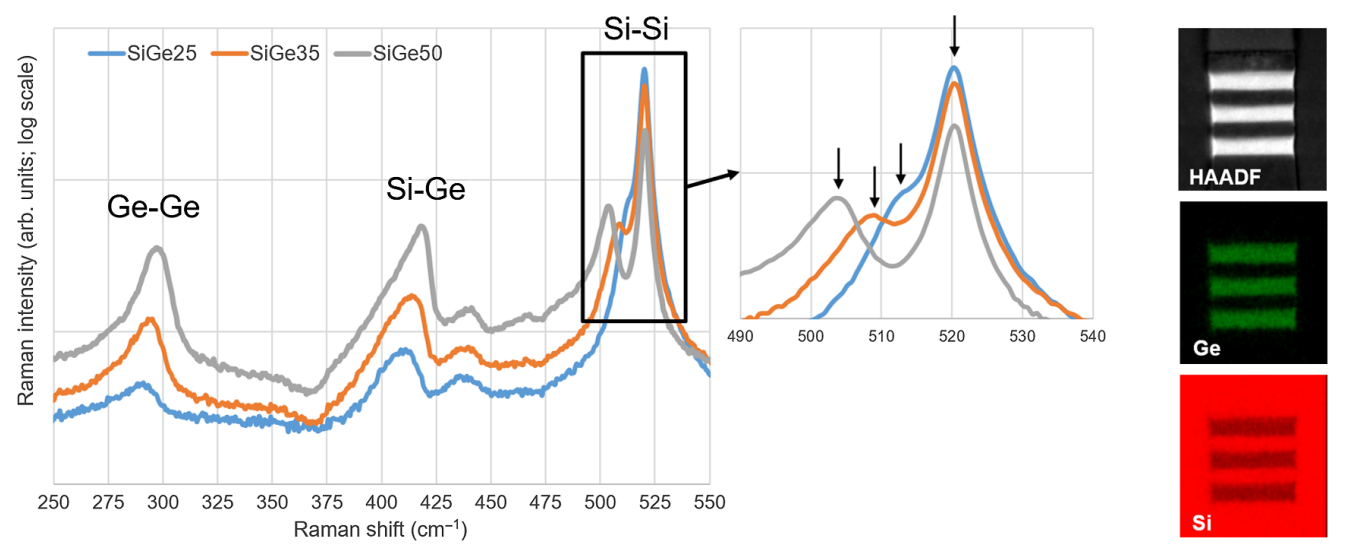

Fig. 2 Raman spectra of the three nanosheet stack samples under investigation $\left(\mathrm{SiGe}_{25}, \mathrm{SiGe}_{35}\right.$, and $\mathrm{SiGe}_{50}$ ) acquired after deposition and before patterning. The images on the right show a representative dark-field cross-section of the $\mathrm{SiGe}_{35}$ after the first patterning step (top) together with qualitative elemental mapping of $\mathrm{Ge}$ (middle) and $\mathrm{Si}$ (bottom). 
spectra shows that the Ge-Ge and the Si-Ge vibrational modes are shifting to higher wavenumbers when the alloy becomes more germanium-rich. The $\mathrm{Si}-\mathrm{Si}$ stretching mode is split and two peaks appear: one at around $520 \mathrm{~cm}^{-1}$ independent of the Ge content and another one below that shifts to lower wavenumbers with increasing Ge content. This behavior is well understood, and several studies have investigated and characterized the mode shifts. ${ }^{16,17}$ The most intense Raman peak at around $520 \mathrm{~cm}^{-1}$ originates from $\mathrm{Si}-\mathrm{Si}$ optic phonons in the strain-free $\mathrm{Si}$ substrate. The second, smaller peak adjacent to it is caused by $\mathrm{Si}-\mathrm{Si}$ stretching motions within $\mathrm{Si}_{(1-x)} \mathrm{Ge}_{x}$ layers and therefore depends on the Ge content $x$.

In addition to composition, the three optic phonon modes $\mathrm{Si}-\mathrm{Si}$, Si-Ge, and $\mathrm{Ge}-\mathrm{Ge}$ also exhibit a characteristic shift in the presence of external strain $\varepsilon$. For this study, composition $x$ and strain $\varepsilon$ of all samples are calculated based on expressions for the experimentally determined peak positions of $\mathrm{Si}-\mathrm{Si}\left(\omega_{\mathrm{Si}-\mathrm{Si}}\right)$ and $\mathrm{Si}-\mathrm{Ge}\left(\omega_{\mathrm{Si}-\mathrm{Ge}}\right)$ published by Tsang et al. ${ }^{16}$ :

$$
\begin{gathered}
\omega_{\mathrm{Si}-\mathrm{Si}}=520.2-62.0 x-815 \varepsilon, \\
\omega_{\mathrm{Si}-\mathrm{Ge}}=400.5+14.2 x-576 \varepsilon .
\end{gathered}
$$

These empirical linear approximations are very good descriptions for samples with a Ge content of $0<x<0.5$. The Ge-Ge mode, the weakest of all three, is not considered for any compositional or strain calculations here. As observed in Fig. 2, and confirmed by Eqs. (1) and $2)$ ), the $\mathrm{Si}-\mathrm{Si}$ and $\mathrm{Si}-\mathrm{Ge}$ phonon-mode shift to lower and higher wavenumbers with increasing $\mathrm{Ge}$ content $x$, respectively. In the case of external strain, both modes red-shift to lower wavenumbers but with different slopes.

\section{Results and Discussion}

\subsection{Comparison to Reference Metrology}

To evaluate the validity of composition and strain results obtained by Raman scattering peak analyses and subsequent calculations with Eqs. (1) and (2), the data can be compared to reference metrology. First, the crystal quality of the non-patterned multilayer stack is evaluated for all wafers by RSMs acquired in the vicinity of the asymmetric (113) Bragg reflection [Figs. 3(a)-3(c)]. The maps confirm that all samples are fully strained as evidenced by the fact that the diffraction peaks from the substrate and the $\mathrm{Si}_{(1-x)} \mathrm{Ge}_{x}$ layers line up in the horizontal direction. Furthermore, the absence of any dislocation scatter around the diffraction peaks from the layers indicates a defect-free pseudomorphic growth. ${ }^{10}$

The sheet-specific Ge content of each wafer is determined by fitting the acquired $\omega-2 \theta$ XRD scans around the (004) Bragg reflection. The average Ge content of all three SiGe sheets is then compared to Raman, which also measures an average of the probed sheets, from the same five locations. The correlation plot of XRD and Raman results for composition shows that both techniques are in excellent agreement with $R^{2}=0.996$ and a slope close to 1 [Fig. 3(d)]. Furthermore, the actual Ge content is close to target. Figure 3(e) shows the correlation plot
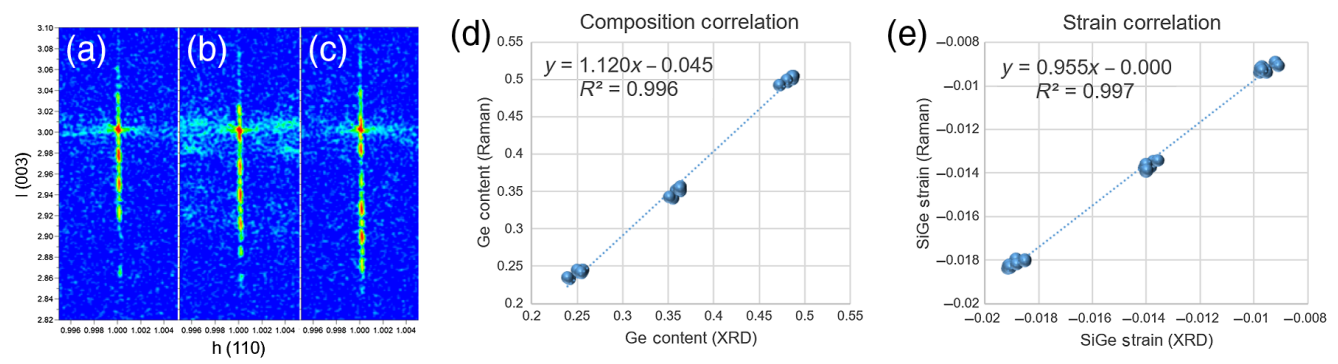

Fig. 3 Measured RSMs in the vicinity of the asymmetric (113) reflection from fully strained, unpatterned nanosheet stacks with (a) $\mathrm{SiGe}_{25}$; (b) $\mathrm{SiGe}_{35}$; and (c) $\mathrm{SiGe}_{50}$ layers; and correlation plots for results from Raman spectroscopy and XRD for (d) composition and (e) strain. 
of the in-plane strain as determined by Raman and XRD. Equally good agreements are observed as for composition. Note that the XRD strain values are calculated, location-specific averages of all three $\mathrm{Si}_{(1-x)} \mathrm{Ge}_{x}$ sheets using the XRD determined Ge content and assuming fully strained layers. Raman results yield a compressive strain of about $-0.9 \%$ for wafers with $x=0.25$ and $-1.8 \%$ for wafers with $x=0.5$. The presented Raman results have been obtained with an excitation wavelength of $405 \mathrm{~nm}$; equally good results are achieved with $457 \mathrm{~nm}$, for example. It is noticeable that the agreement between both techniques for the wafer with the nominally $\mathrm{SiGe}_{50}$ layers is not as good as for the other two wafers with $\mathrm{SiGe}_{25}$ and $\mathrm{SiGe}_{35}$ sheets. This is attributed to the fact that $x=0.5$ is just beyond the validity range of Eq. (2). Specifically, for germaniumrich alloys, the linear approximation used here starts failing to describe the Ge content-dependent Raman shift behavior of the Si-Ge phonon accurately. ${ }^{18}$ Hence, with equations optimized for germanium-rich alloys an accuracy improvement between Raman and XRD can be expected.

Another comparison to reference was done after fin patterning, i.e., after the nanosheet stack is etched to form a line and space grating-like structure. The RSMs in the vicinity of the asymmetric (113) Bragg reflection can be acquired with incident beams parallel and perpendicular to the line and space patterning and allow independent access to properties pertinent to the respective directions. The longitudinal RSMs [not shown for brevity; comparable to Figs. 3(a) and 3(b)] suggest that the in-plane strain along the fins is maintained since the diffraction peaks from the multilayer fin remain accurately aligned with the $\mathrm{Si}$ substrate peak in the horizontal $Q_{x}$ direction. A small shift of the multilayer peaks in the vertical $Q_{z}$ direction towards the $\mathrm{Si}$ substrate peak indicates strain relaxation of the out-of-plane component. ${ }^{19}$ After patterning, there is still no evidence of any dislocation scatter around the diffraction peaks associated with the multilayers. The RSMs measured with an incident beam perpendicular to the fin direction for the three different sample conditions are shown in Fig. 4. In this configuration, the patterning acts as a diffraction grating resulting in multiple diffraction orders (grating rods) along the $Q_{x}$ direction. The distance between the equally spaced grating rods is inversely proportional to the periodicity of the fin grating and a pitch of $100 \mathrm{~nm}$ can be confirmed with an average $\Delta Q_{x}=0.00384{ }^{8,20}$ Also here, there are no indications of non-uniformities: the grating rods and all peaks are well defined and there is no scatter or significant background noise as confirmed also by the corresponding integrated line scans [Figs. 4(d)-4(f)]. Elastic in-plane strain relaxation of the $\mathrm{Si}_{(1-x)} \mathrm{Ge}_{x}$ layers shifts the maximum of the intensity envelope related to the grating rods away from the substrate peak. The reciprocal space position of the grating rods along the $Q_{x}$ direction does not change because the pitch remains unaffected. The intensity envelope maximum can be determined from the integrated line scans. Together with the known Ge concentration $x$ from either XRD at the blanket stage or Raman spectroscopy the transverse component of the in-plane lattice strain can be calculated in a straightforward manner. ${ }^{10}$ The RSM strain results from all
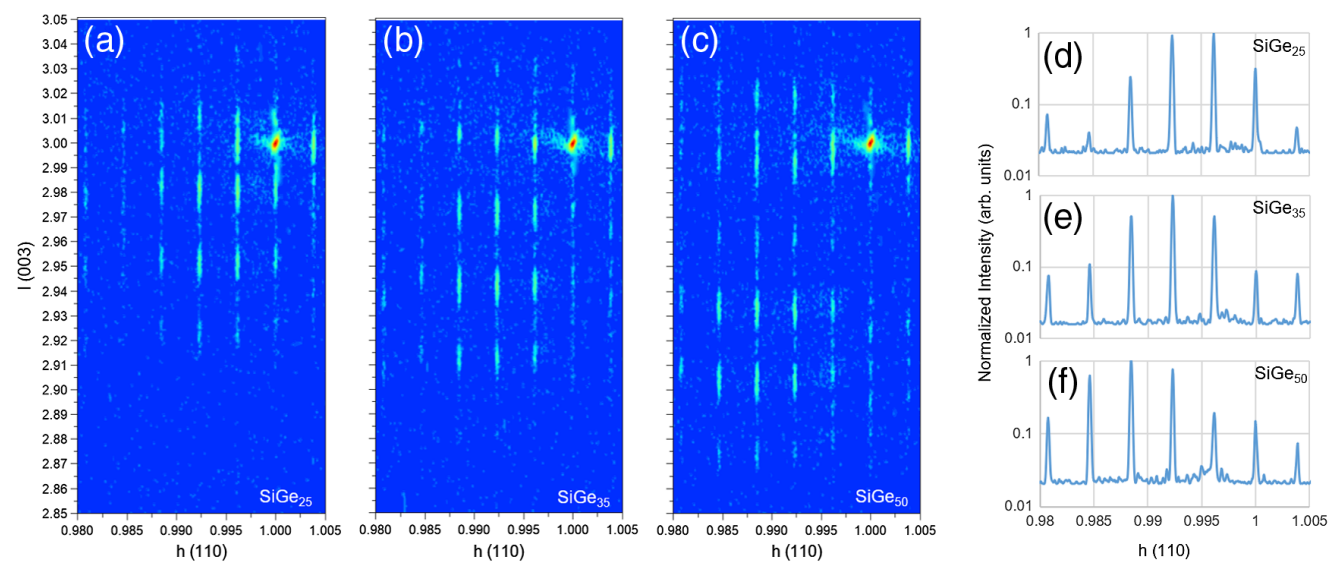

Fig. 4 Measured RSMs in the vicinity of the asymmetric (113) reflection after fin etch (40-nm width at $100-\mathrm{nm}$ pitch) with (a) $\mathrm{SiGe}_{25}$; (b) $\mathrm{SiGe}_{35}$; and (c) $\mathrm{SiGe}_{50}$ layers acquired in the direction perpendicular to the patterning. Corresponding normalized intensity profiles along $h$ for (d) $\mathrm{SiGe}_{25}$ integrated from $I=2.94$ to 2.96 ; (e) $\mathrm{SiGe}_{35}$ integrated from $I=2.930$ to 2.955; and (f) $\mathrm{SiGe}_{50}$ integrated from $I=2.895$ to 2.915 . 

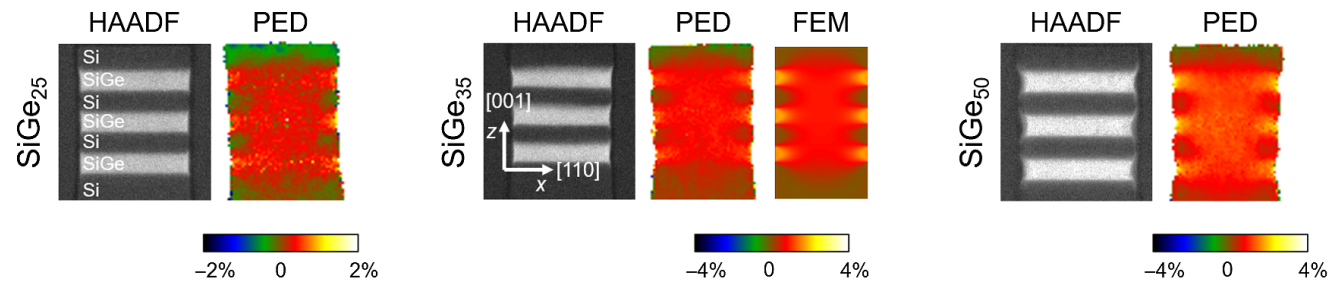

Fig. 5 HAADF STEM and corresponding PED lattice deformation maps for $\mathrm{SiGe}_{25}, \mathrm{SiGe}_{35}$, and $\mathrm{SiGe}_{50}$. The scale bar denotes the lattice deformation with respect to the unstrained Si substrate. The FEM simulation for the $\mathrm{SiGe}_{35}$ sample is also shown for clarity.

three multilayer samples are compared with the strain from the $\mathrm{Si}_{(1-x)} \mathrm{Ge}_{x}$ layers obtained by Raman spectroscopy with polarization perpendicular to the fin direction and shown in Fig. 6 . The transverse strain measurements exhibit an excellent correlation with both $R^{2}$ and slope very close to 1 . The absolute RSM values are slightly lower compared to Raman, which is related to the complex Raman scattering response and a minor influence from phonons coupling to the orthogonal direction.

In addition to the non-destructive RSM strain analysis, a destructive STEM characterization was carried out from fins with a CD of $50 \mathrm{~nm}$. Figure 5 shows cross-fin HAADF images of all three samples together with the corresponding in-plane PED lattice deformation maps. The color coding refers to the lattice parameter change relative to the unstrained Si substrate. It is evident that the patterning leads to a partial relaxation within the $\mathrm{Si}_{(1-x)} \mathrm{Ge}_{x}$ sheets, which is most pronounced at the edges and due to the creation of the unconstraint surfaces. The in-plane deformation is proportional to the $\mathrm{Ge}$ content and most pronounced for the wafer with $\mathrm{SiGe}_{50}$ sheets.

Furthermore, relaxation of the compressively strained $\mathrm{Si}_{(1-x)} \mathrm{Ge}_{x}$ sheets induces a tensile strain within the Si sheets. The finite element method (FEM) simulation depicted for the sample with the $\mathrm{SiGe}_{35}$ sheets is in good agreement with the PED maps and illustrates the abovediscussed behaviors clearly. ${ }^{5,6}$ For this study, no PED lattice deformation maps along the fins were obtained (along the $y$ direction). Previous studies on wafers manufactured in a similar fashion reported that, for practically infinitely long fins, no relaxation occurs and the $\mathrm{Si}_{(1-x)} \mathrm{Ge}_{x}$ sheets remain fully strained. ${ }^{7,19}$ However, simulations for multilayer fins with a length of $500 \mathrm{~nm}$ show some relaxation even along the fins. ${ }^{6}$ Additionally, due to the Poisson effect tensile strain also appears in the $\mathrm{Si}$ sheets along the fins ( $y$ direction), although at lower amounts. ${ }^{5}$ The $y$ direction becomes the channel and hence transport direction, and therefore the induced strain is beneficial for nFET devices since electron mobility is increased in tensile $\mathrm{Si}$.

The calculated average relaxation along the $x$ direction considering the lattice deformation of all three $\mathrm{Si}_{(1-x)} \mathrm{Ge}_{x}$ sheets (average across the entire width) is $40.4 \%, 71.8 \%$, and $75 \%$ for the wafers with $\mathrm{SiGe}_{25}, \mathrm{SiGe}_{35}$, and $\mathrm{SiGe}_{50}$ sheets, respectively. Except for the $\mathrm{SiGe}_{25}$ sample, the relaxations for samples with $\mathrm{SiGe}_{35}$ and $\mathrm{SiGe}_{50}$ sheets are larger than expected, which can be attributed to the destructive sample preparation process and the thinness of the specimen. For example, nanobeam diffraction studies have shown that strain relaxation originating from a fin edge can still affect the lattice more than $100 \mathrm{~nm}$ away from that unconstraint surface. ${ }^{19}$ Previously, it was assumed that the complex response from transverse Raman scattering may be a measure of the average in-plane strain along the $x$ and $y$ directions, which matched the calculated in-plane average from PED analyses. ${ }^{21}$ However, simulations and the excellent correlation between Raman and RSM shown in Fig. 6 suggest that the Raman scattering observed with polarization perpendicular to the grating-like pattern is strongly dominated by the transverse properties along the $x$ direction. To minimize the influence of a potential relaxation caused by the destructive sample preparation, lattice deformation read-out was limited to center of the first $\mathrm{Si}_{(1-x)} \mathrm{Ge}_{x}$ sheet (average of 20 pixels) as the bottom part is least affected from edge relaxation. ${ }^{19}$ The correlation between computed transverse PED in-plane strain and corresponding transverse Raman spectroscopy results is shown in Fig. 6. While the $R^{2}=0.95$ confirms a very good linear behavior, the slope of $<1$ reveals some discrepancies. For $\mathrm{SiGe}_{25}$ sheets, both techniques are in excellent agreement with a compressive strain of $\sim-0.006$. However, with increasing germanium concentration, PED results suggest less strain and hence more relaxation 


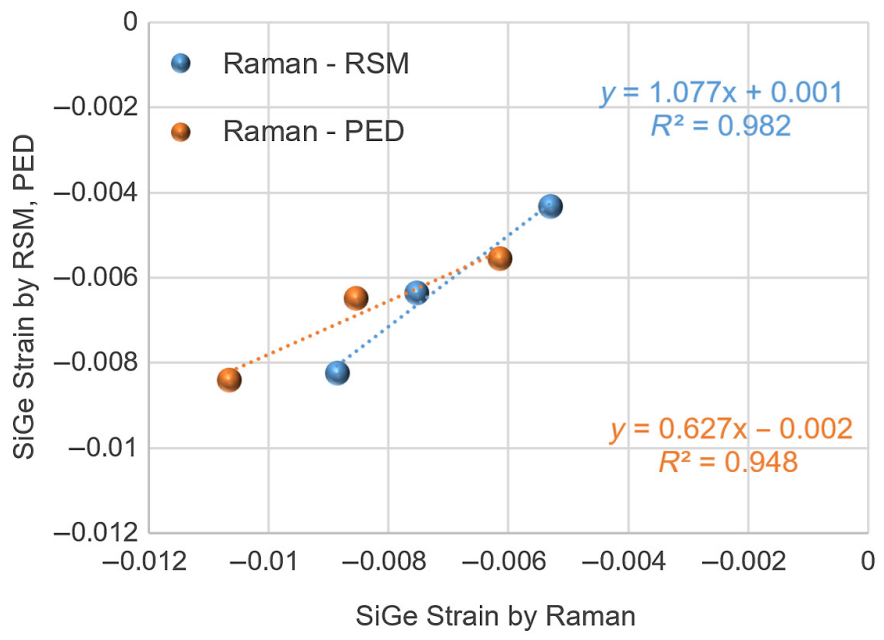

Fig. 6 Transverse in-plane strain as obtained by Raman, RSMs, and PED. The RSMs and PED results are from fins with a width of 40 and $50 \mathrm{~nm}$, respectively, and compared to Raman results from corresponding fin dimensions. The Raman results represent the average strain of the probed $\mathrm{Si}_{(1-x)} \mathrm{Ge}_{x}$ layers as obtained from a measurement with polarization perpendicular to the fin direction. The transverse RSM results represent the average response from the $\mathrm{Si}_{(1-x)} \mathrm{Ge}_{x}$ layers within multilayer fin pattern. The PED results are obtained by averaging a region of interest $\left(10 \times 2\right.$ pixels) from the center of the first $\mathrm{Si}_{(1-x)} \mathrm{Ge}_{x}$ sheet.

in the $\mathrm{Si}_{(1-x)} \mathrm{Ge}_{x}$ sheets compared to Raman. As discussed above, this is mainly attributed to the destructive sample preparation procedure and the thinness of the specimen leading to additional relaxation.

\subsection{Raman Strain Monitoring}

With the assurance that strain results obtained by Raman spectroscopy are in good agreement with reference metrology for blanket and patterned targets, all presented strain results from here on are from Raman metrology only. Figure 7(a) shows the compressive strain in $\mathrm{Si}_{(1-x)} \mathrm{Ge}_{x}$ sheets for all three samples as a function of all 21 dies measured across the wafer. The four graphs correspond to measurements of an unpatterned blanket target and three-patterned targets with different fin CDs $(40,50$, and $70 \mathrm{~nm})$. A representative cross-section of the patterned target with 40-nm fin CD is also depicted [inset in Fig. 7(b)]. As discussed already before, the strain increases with Ge content due to the increasing lattice mismatch. The consistent measurements
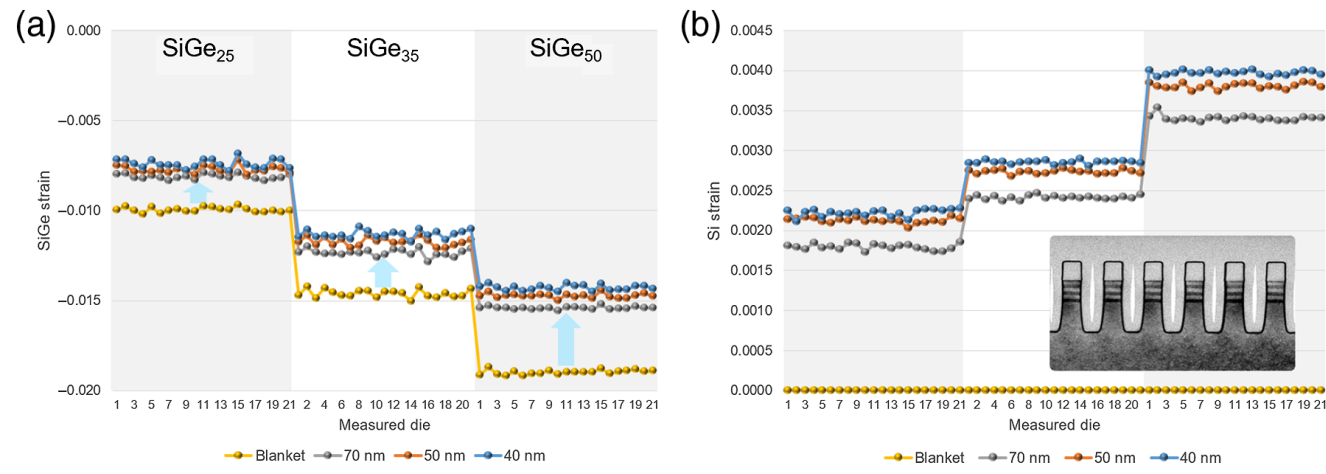

Fig. $7 \mathrm{Si}_{(1-x)} \mathrm{Ge}_{x}(\mathrm{a})$ and $\mathrm{Si}(\mathrm{b})$ strain for blanket and three different fin CDs (40, 50, and $\left.70 \mathrm{~nm}\right)$ as a function of measured dies across the wafer. The three vertical sections in (a) and (b) represent the three different samples with $\mathrm{SiGe}_{25}, \mathrm{SiGe}_{35}, \mathrm{SiGe}_{50}$ sheets. The inset in (b) shows a STEM cross-section of the $\mathrm{SiGe}_{25}$ sample with $40-\mathrm{nm}$ fin $\mathrm{CD}$. All results are obtained with polarization along the fin direction. 
across the wafer indicate that metrology noise and processing variations are very low, and no across wafer signature is observed. The average across wafer Ge content determined by Raman is $25.2 \%, 37.2 \%$, and $49.2 \%$ for the nominally $\mathrm{SiGe}_{25}, \mathrm{SiGe}_{35}$, and $\mathrm{SiGe}_{50}$ samples, respectively. With patterning and the creation of unconstraint surfaces, relaxation occurs, which depends on the fin $\mathrm{CD}$, i.e., with smaller CDs greater relaxation is observed. This can be explained with the increasing surface-to-volume ratio with decreasing CDs and hence the increasing dominance of the strong edge relaxation. Across the three samples, the average measured relaxation with polarization along the fins is approximately constant with $19 \%, 22 \%$, and $25 \%$ for the $70-$, $50-$, and 40-nm features, respectively. It is noteworthy that long multilayer fins (from $\sim 300 \mathrm{~nm}$ away from a fin end ${ }^{19}$ ) remain fully strained along the fin direction, which is also confirmed by the RSM analyses. Hence, the Raman scattering response with polarization parallel to the fin direction comprises contributions from both in-plane orientations. Strongly dominated by the LO phonon mode, it is approximately a measure of the average in-plane relaxation.

Figure 7(b) shows the same graph but for tensile Si sheets. It is noteworthy that at the unpatterned blanket stage, the Si sheets do not exhibit any strain. In this case, the Si-Si LO-TO Raman phonon of the unstrained Si sheets is identical to the phonon mode of the strain-free Si substrate. Hence the simulated data points at zero strain are not measurements and only added for convenience. Once the blanket multilayer stack is patterned and the strained $\mathrm{Si}_{(1-x)} \mathrm{Ge}_{x}$ relaxes, a tensile strain is induced in the $\mathrm{Si}$ sheets. The tensile strain in Si depends on the Ge content within the $\mathrm{Si}_{(1-x)} \mathrm{Ge}_{x}$ and on the sheet CD. The Si strain for the wafer with the $\mathrm{SiGe}_{50}$ sheets is about a factor of two larger compared to the multilayer stacks with $\mathrm{SiGe}_{25}$ sheets. Furthermore, the sheets with the smallest $C D(40 \mathrm{~nm})$ exhibit the largest strain and there is a linear relationship between strain and $\mathrm{CD}$ within the measured range.

As discussed earlier already, there is a directional strain dependence post fin patterning, which can be distinguished based on the polarization direction of the probing light. Polarization-dependent results of the compressive strain in $\mathrm{Si}_{(1-x)} \mathrm{Ge}_{x}$ after fin patterning are shown in Fig. 8. The box chart is vertically sectioned accommodating the three sample scenarios and the $x$ axis sorts the four targets by increasing CD (the unpatterned blanket can be understood as having an infinitely large CD). The blanket strain probed with parallel and perpendicular polarization states is practically identical. This is expected, as the Raman peak position for two orthogonal directions from a Si $(100)$ wafer is identical. ${ }^{14}$ The patterned sites on the other hand

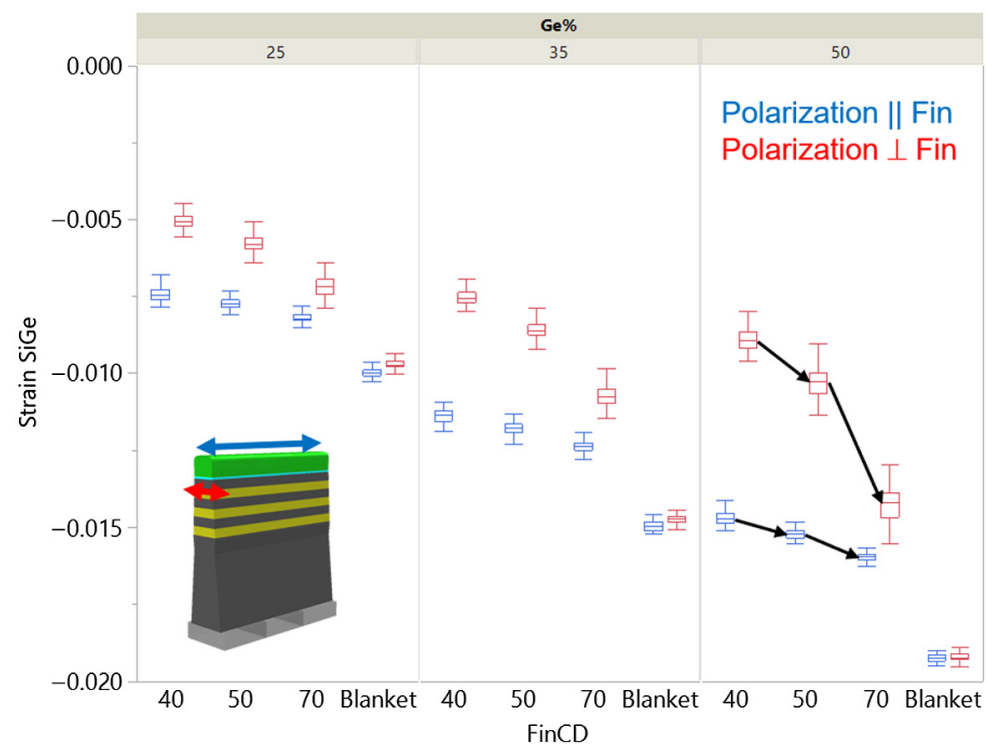

Fig. 8 Polarization dependence of $\mathrm{Si}_{(1-x)} \mathrm{Ge}_{x}$ strain results post fin patterning. The chart is split into three vertical sections representing the three sample types with different Ge content under investigation. The $x$ axis within each section groups the results by fin CD and includes the unpatterned blanket site. The blue and red boxes denote the results for polarization parallel and perpendicular to the fins, respectively. The black arrows are merely a guide to the eye. 
exhibit a strong polarization-dependent strain difference. When probing the strain with polarization parallel to the fins, the value is larger compared to a polarization perpendicular to the fins. This general observation is in agreement with previously published results and corresponding simulations. ${ }^{5,6,8}$ Furthermore, the compressive strain difference increases significantly with decreasing $\mathrm{CD}$, and the measurement with perpendicular polarization is more sensitive to $\mathrm{CD}$ changes. In general, the trends for the wafers with different Ge concentrations are comparable but differ in amplitude.

Similar to the size-dependent strain behavior along the fin direction, a linear relationship between transverse strain and width is observed within the measured range. The experimentally determined relaxation of $49 \%$ for multilayer $\mathrm{SiGe}_{35}$ fins with a $40-\mathrm{nm} \mathrm{CD}$ is already in good agreement with a reported simulated strain relaxation of $58 \%$ for similar fins with a $35-\mathrm{nm} \mathrm{CD}{ }^{6}$ An extrapolation beyond the measured CD range toward fins with a width of $35 \mathrm{~nm}$ for the sample with $\mathrm{SiGe}_{35}$ comes with $52.5 \%$ relaxation even closer to the literature value.

While this paper only focuses on strain characterization, which is based on the determination of the phonon mode energy, it should be noted that Raman intensities (phonon-mode amplitude) may be used to measure the $\mathrm{CD} .^{22}$

\subsection{Strain Tracking Through FEOL Patterning}

Figure 9 shows the $\mathrm{SiGe}_{25}$ strain evolution through FEOL patterning after six important processing steps: blanket (nanosheet stack deposition), FinEtch (fin patterning along $y$; "nanosheet stack lines"), GateEtch (dummy gate patterning), FinRecess (fin patterning along $x$; "nanosheet stack pillars"), S/D epi (Si:P epitaxy), and PolyPull (dummy gate removal). The five insets at the top schematically depict the structure at the respective processing step with an orientation parallel to the original fin direction (channel direction). PolyPull is the last opportunity to measure the sacrificial $\mathrm{Si}_{(1-x)} \mathrm{Ge}_{x}$ sheets as they get removed at the next processing step. Focus is put on nFET devices and polarization along the $y$ direction (original fin direction). The boxes and whiskers represent measured values for all fin and gate CDs and indicate the across wafer variation convoluted with some metrology uncertainty.

As discussed before, processing starts with fully strained $\mathrm{SiGe}_{25}$ layers at the blanket stage due to a defect-free pseudomorphic growth. After the first patterning step (FinEtch) already the most significant impact on strain is observed with on average $>20 \%$ relaxation. At GateEtch a small relaxation is observed, which is mostly attributed to several thermal processing steps

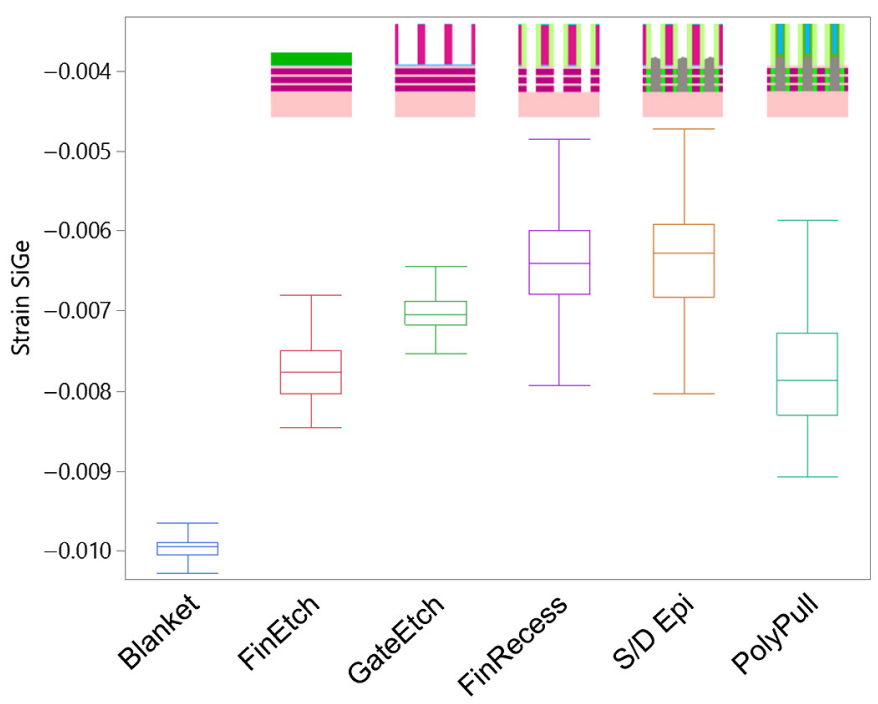

Fig. $9 \mathrm{SiGe}_{25}$ strain evolution through FEOL patterning measured after six processing steps. Results depicted are for nFET devices and polarization along the $y$ direction. The insets at the top schematically depict the structure at the respective processing step with a view parallel to the $y$ direction (along the fins). 
within the fin module. It is noteworthy that at GateEtch the major contribution to the Raman response is from the exposed fin areas not covered by the dummy gate. The next step is FinRecess, which is a fin patterning perpendicular to the original fin direction defining the gate length. This leaves nanosheet stack pillars with dimensions of the fin $\mathrm{CD}$ in one direction and dummy gate $\mathrm{CD}$ in the other direction. On average, some further relaxation is observed, which is due to the creation of more unconstraint surfaces. Additionally, an increase in the data spread is noticed and mainly caused by the influence of the now present and varying gate $\mathrm{CD}$. Similar to previously discussed trends related to the fin $\mathrm{CD}$, with decreasing gate $\mathrm{CD}$ the sheet relaxation increases but not as dramatic as compared to what was measured at FinEtch. Nonetheless, the gate CD-dependent strain behavior does not explain all observed variation. The reduced amount of sheet volume together with the polycrystalline dummy gate, which causes a broad Raman background thereby contributing to measurement uncertainty, and likely some process-related variations add to the overall data spread. It is noteworthy that light needs to pass through the polycrystalline dummy gate twice before Raman scattering can be collected.

For this wafer, the strain remains approximately constant after S/D epitaxy with an equally large distribution. After PolyPull, when all polycrystalline dummy gate material is removed, an increased compressive $\mathrm{SiGe}_{25}$ strain is measured again. Further studies also supported by strain modeling are required to fully understand what is happening. Possibilities may be related to dummy gate clamping, S/D epi strain, processing, or strain modeling inaccuracies due to the polycrystalline material at the previous steps.

Figure 10(a) shows the Si tensile strain evolution through FEOL patterning after six important processing steps: FinEtch, GateEtch, FinRecess, S/D epi, PolyPull, and Release. The latter step refers to the removal of the sacrificial $\mathrm{SiGe}_{25}$ sheets. At each step, the strain is plotted for all three fin CDs. The boxes and whiskers represent all measured values and the across wafer variation convoluted with some metrology uncertainty. It is noteworthy that the blanket step is not included because the Si sheets do not exhibit any strain before patterning. The results at FinEtch have been discussed in detail already above [Fig. 7(b)]; briefly, CD-dependent tensile strain is generated by the $\mathrm{SiGe}_{25}$ relaxation. It is noticeable at GateEtch that the difference between the three fin CDs is less pronounced as compared to FinEtch and mainly related to the strain decrease of the 40 and $50 \mathrm{~nm}$ device structures. This may be caused by thermal processing in the fin module. At FinRecess, there is a small increase in strain due to further edge relaxation of the $\mathrm{SiGe}_{25}$ sheets along with a significant increase in the data spread. This data spread increase was also observed for the $\mathrm{SiGe}_{25}$ strain at the same process step and is related to the different gate CDs with additional contributions from possible process variations and an increased measurement uncertainty due to the smaller Si sheet area located underneath the thick polycrystalline gates. A substantial increase post S/D epitaxy is observed with no major strain changes transitioning to PolyPull. After removal of the polycrystalline dummy gate material the data spread

(a)

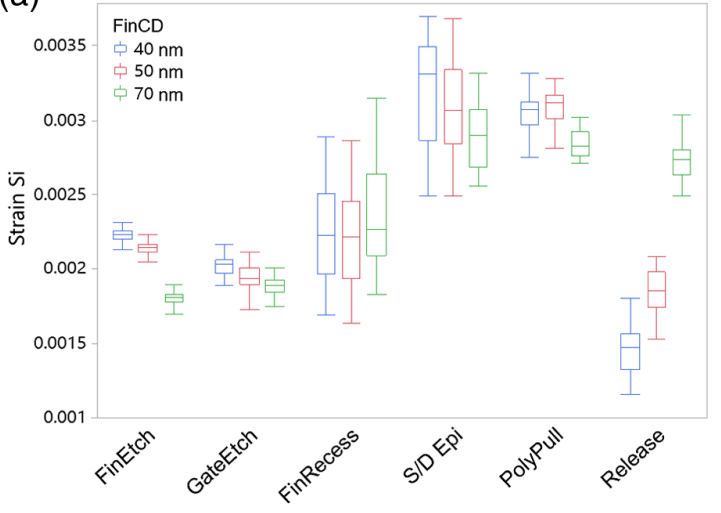

(b)

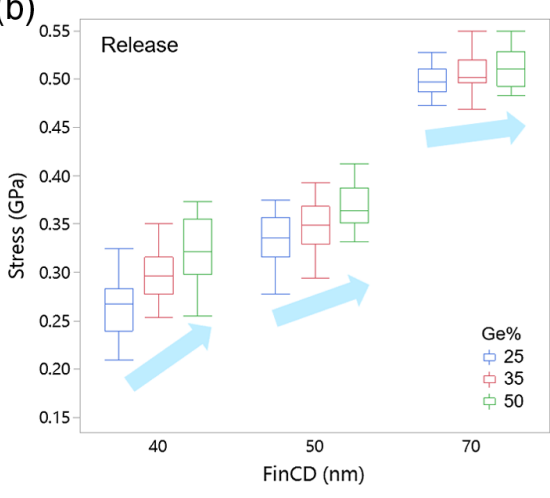

Fig. 10 (a) Tensile Si strain evolution through FEOL patterning measured after six processing steps, where each box per step represents a different fin CD. Results depicted are for nFET devices with $\mathrm{SiGe}_{25}$ sheets; (b) Si stress post channel release as a function of fin $\mathrm{CD}$ and $\mathrm{Ge} \%$. Only strain and stress values from measurements with polarization along the $y$ direction are compared. 
decreases again slightly. It is noteworthy that since the measured devices are nFETs, the S/D and the channel are comprised of epitaxial $\mathrm{Si}$. It has been confirmed that the selected experimental settings allow for probing the Si nanosheet channels only, independent of the S/D region. Choosing a different measurement configuration, it is possible to probe the S/D epitaxial Si: P only.

After the complete removal of the sacrificial $\mathrm{SiGe}_{25}$ nanosheets (Release), a significant sizedependent strain relaxation is observed. However, the Si nanosheets still remain under tensile strain. Now, the smallest fin CD exhibits also the smallest tensile strain and with increasing CD the strain increases substantially. This trend is opposite to what was observed at FinEtch and likely related to edge relaxation, which is more pronounced when the surface-to-volume ratio is large. The gate CD plays a minor role only at PolyPull and Release and is not discussed.

A more detailed analysis after the Release step is shown in Fig. 10(b). The Si strain was converted to stress in GPa using a Young's modulus $E=180 \mathrm{GPa}$. Besides looking at CDdependent stress behavior, results from the wafers with different Ge content are presented. For each measured CD, the stress increases with Ge content. This means that strain tuning of the $\mathrm{Si}$ channel is possible by variation of the Ge content in the sacrificial $\mathrm{Si}_{(1-x)} \mathrm{Ge}_{x}$ nanosheets.

Most observed trends are in agreement with previously published simulations by Reboh et al. ${ }^{6}$ Specifically, the emergence of tensile strain after fin patterning and the fact that tensile strain post channel release depends on the Ge content of the sacrificial $\mathrm{Si}_{(1-x)} \mathrm{Ge}_{x}$ nanosheets. Notably, simulations suggest an average stress of $\sim 0.3 \mathrm{GPa}$ for devices using sacrificial $\mathrm{SiGe}_{30}$, which is close to what is measured by Raman spectroscopy on wafer.

\section{Conclusions and Outlook}

In-line Raman spectroscopy for $\mathrm{Si}$ and $\mathrm{Si}_{(1-x)} \mathrm{Ge}_{x}$ nanosheet strain metrology throughout the FEOL manufacturing cycle of next-generation GAA nanosheet field-effect transistors was presented and results discussed. Three samples with intentional strain variations, by changing the Ge content $(x=0.25,0.35$, and 0.50$)$ of the pseudomorphic $\mathrm{Si}_{(1-x)} \mathrm{Ge}_{x}$ nanosheets, were manufactured and measured at seven critical processing steps using polarization-dependent in-line Raman spectroscopy. To characterize and quantify the strain evolution, the precise position of the $\mathrm{Si}-\mathrm{Si}$ and $\mathrm{Si}-\mathrm{Ge}$ optical phonon modes was determined at each process step, based on which composition and strain can be calculated.

The importance of excitation wavelength was discussed with regards to penetration depths and scattering cross-sections. It was highlighted how critical polarization-dependent measurements are for blanket films and particularly when evaluating patterned anisotropic samples. Especially when characterizing orientation-dependent strain or when epitaxial Si in channel and source/drain needs to be characterized independently, for instance.

The accuracy of the Raman metrology was confirmed with reference data for composition and strain obtained after nanosheet stack epitaxy on unpatterned targets by non-destructive highresolution x-ray diffraction. It was found that both methodologies are in excellent agreement. Additionally, RSMs as well as destructive lattice deformation maps determined from PED after the first line and space patterning were acquired to calculate strain. A comparison to Raman spectroscopy revealed a very good agreement and supported the understanding of polarization-dependent settings. It was demonstrated that polarization-dependent laser excitations parallel and perpendicular to patterned fins allow for probing different parts of the in-plane strain components. An experiment with polarization perpendicular to the fins yields a response strongly dominated by the relaxation pertinent to that direction, while a polarization parallel to the fins results approximately in an average in-plane relaxation. The observed trends agree well with reference measurements and previously published simulations. ${ }^{5,6}$

Monitoring the strain evolution of Si sheets through patterning processes, it was found that the experimentally determined results from in-line Raman spectroscopy follow trends of previously published simulations. ${ }^{6}$ Specifically, a successively increasing relaxation of the compressive $\mathrm{Si}_{(1-x)} \mathrm{Ge}_{x}$ strain after FinEtch (nanosheet stack lines) and FinRecess (nanosheet stack

pillars) induces an increasing tensile strain in the Si sheets. The strain is strongly size-dependent, and the tensile Si sheet strain can be engineered by Ge content variation of the sacrificial layers. 
The absolute value of the calculated Si nanosheet stress post channel release agrees with FEM simulations.

The presented in-line Raman metrology is developed for non-destructive nFET device baseline monitoring. Even more important is strain metrology for pFET devices particularly because complex strain engineering approaches are developed to boost hole mobility. ${ }^{4}$ While the electron mobility in nanosheet devices is much better compared to finFETs due to the channel orientation and the intrinsic tensile strain induced by the sacrificial sheets, the hole mobility in the same channels is rather low. Therefore, compressive $\mathrm{Si}_{(1-x)} \mathrm{Ge}_{x}$ channels are desired. Front-up dual processing is complex and costly, hence a iGe channel last approach has been proposed recently. ${ }^{4}$ The innovative part starts post $\mathrm{Si}_{(1-x)} \mathrm{Ge}_{x}$ channel release and the method relies on trimming down the $\mathrm{Si}$ channels to a thickness of about $2 \mathrm{~nm}$ before growing a pseudomorphic $\mathrm{Si}_{(1-x)} \mathrm{Ge}_{x}$ cladding all around the channel. In-line strain monitoring with Raman spectroscopy at multiple processing steps within this flow (e.g., after trimming and after $\mathrm{Si}_{(1-x)} \mathrm{Ge}_{x}$ growth) will be an invaluable addition to ensure successful development and consistent high volume manufacturing of these next-generation GAA nanosheet devices.

\section{Acknowledgments}

The authors would like to thank Marjorie Cheng, Jennifer Fullam, Manasa Medikonda, Roger Cornell, Gilad Barak, John Gaudiello, Raja Muthinti, Michael Sendler, Mary Breton, Nelson Felix, and Bala Haran for support of this work.

\section{References}

1. M. Lapedus, "New transistor structures At 3 nm/2 nm," 2021, https://semiengineering.com/ new-transistor-structures-at-3nm-2nm/ (2 February 2021).

2. N. Loubet et al., "Stacked nanosheet gate-all-around transistor to enable scaling beyond FinFET," in Symp. VLSI Tech., 230-231 (2017).

3. G. Tsutsui et al., "Strain engineering in functional materials," AIP Adv 9, 030701 (2019).

4. S. Mochizuki et al., "Stacked gate-all-around nanosheet PFET with highly compressive strained SiGe channel," in IEEE Int. Electron Dev. Meeting (2020).

5. S. Reboh et al., "Imaging, modeling and engineering of strain in gate-all-around nanosheet transitors," in IEEE Int. Electron Dev. Meeting (2019).

6. S. Reboh et al., "Strain, stress, and mechanical relaxation in fin-patterned Si/SiGe multilayers for sub-7 nm nanosheet gate-all-around device technology," Appl. Phys. Lett. 112, 051901 (2018).

7. J. Li et al., "Precession electron diffraction (PED) strain measurements in stacked nanosheet structures," Microsc. Microanal. 25(Suppl. 2), 2018 (2019).

8. A. Schulze et al., "Strain and compositional analysis of (Si)Ge fin structures using high resolution x-ray diffraction," Phys. Status Solidi C 14, 1700156 (2017).

9. D. De Salvador et al., "Lattice parameter of Si1-x-yGexCy alloys," Phys. Rev. B 61, 13005 (2000).

10. Z. Ma and D. G. Seiler, Metrology and Diagnostic Techniques for Nanoelectronics, Pan Stanford, Singapore (2017).

11. I. De Wolf, "Micro-Raman spectroscopy to study local mechanical stress in silicon integrated circuits," Semicond. Sci. Technol. 11, 139 (1996).

12. T. Nuytten et al., "Edge-enhanced Raman scattering in narrows Ge fin field-effect transistor channels," Appl. Phys. Lett. 106, 033107 (2015).

13. A. Picco et al., "Raman efficiency in SiGe alloys," Phys. Rev. B 82, 115317 (2010).

14. W. S. Yoo, H. Harima, and M. Yoshimoto, "Polarized Raman signals from Si wafers: dependence of in-plane incident orientation of probing light," ECS J. Solid State Sc. 4, P356 (2015).

15. V. Poborchii, T. Tada, and T. Kanayama, "Edge-enhanced Raman scattering in Si nanostripes," Appl. Phys. Lett. 94, 131907 (2009). 
16. J. C. Tsang et al., "Measurements of alloy composition and strain in thin $\mathrm{GexSi}_{1-x}$ layers," J. Appl. Phys. 75, 8098 (1994).

17. H. Rücker and M. Methfessel, "Anharmonic Keating model for group-IV semiconductors with application to the lattice dynamics in alloys of Si, Ge, and C," Phys. Rev. B 52, 11059 (1995).

18. M. I. Alonso and K. Winer, "Raman spectra of c-Si ${ }_{1-x} \mathrm{Ge}_{x}$ alloys," Phys. Rev. B 39, 10056 (1989).

19. S. Mochizuki et al., "Quantification of local strain distributions in nanoscale strained SiGe FinFET structures," J. Appl. Phys. 122, 135705 (2017).

20. P. Y. Hung et al., "Application of inline high resolution x-ray diffraction in monitoring Si/ SiGe and conventional Si in SOI fin-shaped field effect transistor processes," J. Vac. Sci. Technol. B 30, 041211 (2012).

21. D. Schmidt et al., "In-line Raman spectroscopy for stacked nanosheet device manufacturing," Proc. SPIE 11611, $116111 \mathrm{~T}$ (2021).

22. A. Gawlik et al., "Critical dimension metrology using Raman spectroscopy," Appl. Phys. Lett. 117, 043102 (2020).

Biographies of the authors are not available. 